Article

\title{
Diurnal Expression of the Per2 Gene and Protein in the Lateral Habenular Nucleus
}

\author{
Zhigong Zhao, Haiyan Xu, Yongmao Liu, Li Mu, Jinyu Xiao and Hua Zhao * \\ Department of Physiology, College of Basic Medical Sciences, Jilin University, 126 Xinmin Street, \\ Changchun 130021, China; E-Mails: studayhard518@sina.com (Z.Z.); hyxu@jlu.edu.cn (H.X.); \\ lymmm8338@sina.com (Y.L.); muli_xf@126.com (L.M.); zacshaw@outlook.com (J.X.) \\ * Author to whom correspondence should be addressed; E-Mail: zhua@jlu.edu.cn; \\ Tel.: +86-431-8561-9472; Fax: +86-431-8563-9362.
}

Academic Editor: Kenji Hashimoto

Received: 22 May 2015 / Accepted: 20 July 2015 / Published: 23 July 2015

\begin{abstract}
The suprachiasmatic nucleus plays an important role in generating circadian rhythms in mammals. The lateral habenular nucleus $(\mathrm{LHb})$ is closely linked to this structure. Interestingly, the $\mathrm{LHb}$ shows a rhythmic firing rate in vivo and in vitro, and sustained oscillation of rhythmic genes in vitro. However, under the in vivo condition, whether rhythmic gene expression in the LHb has circadian rhythms remains unknown. In this study, we examined LHb tissue in rats to determine Period2 (Per2) gene and protein expression at six zeitgeber time points (ZT2, ZT6, ZT10, ZT14, ZT18, and ZT22) in a 12-h light and 12-h dark (LD) environment. We found that in the LD environment, Per 2 gene expression and PER2 protein levels in the LHb were higher in the day and lower in the night, showing periodic oscillation, with a peak at ZT10 and a trough at ZT22 (Per2 mRNA) and ZT18 (PER2 protein). We conclude that Per2 expression and PER2 protein levels in the LHb have rhythmic oscillation in vivo. This study provides a basis for further study on the role of the LHb in the circadian rhythm system.
\end{abstract}

Keywords: period2 gene; circadian rhythm; lateral habenular nucleus; suprachiasmatic nucleus; running-wheel activity 


\section{Introduction}

Circadian rhythms are observed in all physiological and behavioral processes of mammals. The suprachiasmatic nucleus ( $\mathrm{SCN}$ ) of the anterior hypothalamus is the major pacemaker of the system for generating and sustaining daily circadian rhythms in mammals [1-3]. The daily physiological and behavioral circadian rhythms in rats disappear after the SCN is lesioned bilaterally [1,4]. The SCN generates internal circadian rhythms in many behavioral and physiological functions, and also synchronizes them to environmental light-dark cycles via the direct retinohypothalamic tract [5-7]. Therefore, many researchers have focused on the role of the SCN in investigating the regulatory mechanisms of circadian rhythms. However, several studies have raised the possibility that some circadian oscillators of varying strength exist in other brain areas in addition to the SCN [8-10]. This indicates that circadian processes in the brain are not solely influenced by the SCN. These nuclei may play an important role in linking SCN output to the system in vivo. Therefore, such extra-SCN sites need to be identified to reveal the mechanisms of circadian rhythms.

One of the structures with rhythmicity is the habenular nucleus ( $\mathrm{Hb})$, particularly the lateral habenular nucleus ( $\mathrm{LHb}$ ), which plays an important role in conveying limbic forebrain input to mid-brain structures [11-13]. The LHb is involved in a wide range of functions, such as stress responses, reproductive behavior, and sleep, which are regulated on a circadian basis [14-16]. Some of the affective disorder diseases, which are attributed to the LHb, are related to disrupted circadian rhythms [17,18]. Recently, the LHb structure was suggested as a component of the brain's circadian system $[19,20]$. The roles of the LHb in physiological function and disease may be associated with its own rhythmic activities and changes, and may convey the information from the SCN to systems controlling biological circadian rhythms [9,21].

Our previous study showed that the firing rates of rat $\mathrm{Hb}$ neurons were altered in response to retinal illumination in vivo. In addition, LHb neuronal activity showed rhythmic oscillation in in vivo and in vitro experiments [22]. Tavakoli-Nezhad reported that the expression of c-Fos protein in the medial division of the LHb showed a rhythmic oscillation in hamsters [23]. Recent research has shown that the transecting fasciculus retroflexus, which is the major efferent pathway of the LHb, alters the daily amount of motor activity and extends the circadian rest-activity rhythm cycle set by the SCN [20]. Circadian oscillators of clock gene expression are present in the LHb in vitro [24]. In addition, the LHb receives a vasopressin-containing projection from the SCN [25]. Taken together, these findings suggest that the LHb may be associated with the circadian rhythm system as a downstream structure of the SCN. However, whether rhythmic clock gene/protein expression exists in the LHb in vivo and whether it shows zeitgeber time-keeping properties in this structure are unknown.

To address these issues, this study aimed to investigate Period2 (Per2) gene expression and PER2 protein levels under 12-h light and 12-h dark (LD) conditions at different time points to identify the diurnal oscillation of LHb activity in vivo in rats. 


\section{Results and Discussion}

\subsection{Expression of Per2 $m R N A$ in the $L H b$ of $L D$ Rats}

We determined six zeitgeber time points (ZT2, ZT6, ZT10, ZT14, ZT18, ZT22) in a LD environment to examine diurnal Per2 mRNA expression in the LHb. Multigroup data in the time series showed significant differences by one-way analysis of variance (ANOVA) $(\mathrm{F}[5,30]=21.659, p<0.01)$. The post hoc analysis was then conducted to compare the differences of every two time points. We found that Per2 expression peaked at ZT10 and was lowest at ZT22. Per2 expression at ZT6 was also high, similar to that at ZT10. Per2 expression at ZT6 and ZT10 was significantly higher than that at ZT2, ZT14, ZT18 and ZT22 (all at $p<0.01$ ). Per2 expression at ZT2 was significantly higher than that at ZT18 $(p<0.05)$ and ZT22 $(p<0.01)$ (Figure 1).

$\mathbf{A}$

$\begin{array}{llllll}\text { ZT2 } & \text { ZT6 } & \text { ZT10 } & \text { ZT14 } & \text { ZT18 } & \text { ZT22 }\end{array}$

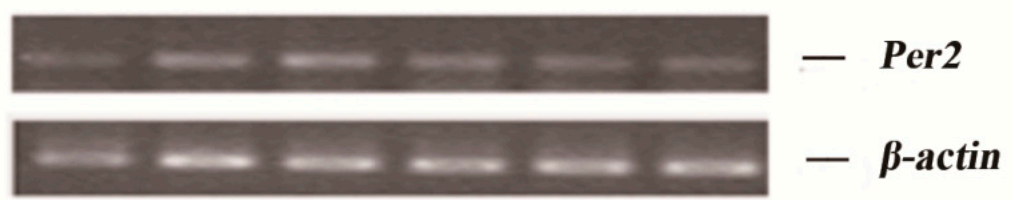

B

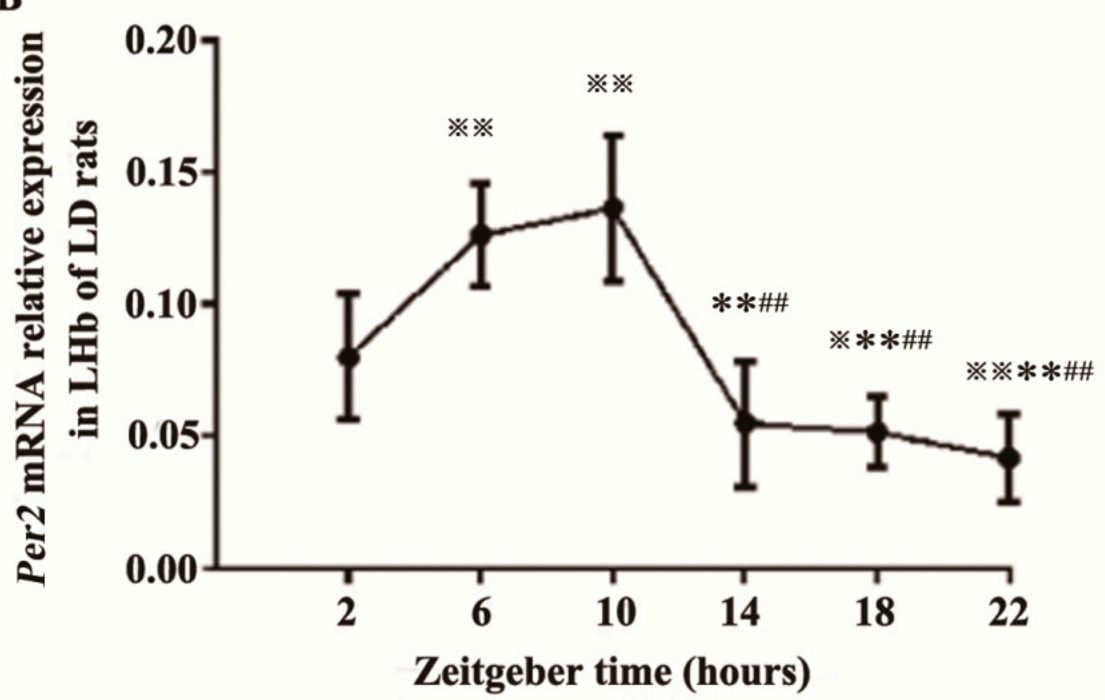

Figure 1. Rhythmic expression of Per2 mRNA in the lateral habenular nucleus (LHb) of LD rats. (A) Representative gel electrophoresis of real-time polymerase chain reaction products stained with ethidium bromide (EB), showing Per2 and $\beta$-actin mRNA in the LHb at six ZT time points; and (B) Relative quantitative analysis of Per $2 \mathrm{mRNA}$ in the $\mathrm{LHb}$ at six ZT time points. All values are normalized against the housekeeping gene $\beta$-actin. Data are presented as mean $\pm \operatorname{SD}(n=6)$. ${ }^{*} p<0.05$ and ${ }^{* *} p<0.01$ compared with ZT2 time point; ** $p<0.01$, compared with ZT6 time point; ${ }^{\# \#} p<0.01$, compared with ZT10 time point. 


\subsection{Expression of PER2 Protein in the LHb of LD Rats}

PER2 relative protein expression at six zeitgeber time points was determined. There was significant differences in multigroup data of the time series by one-way ANOVA $(\mathrm{F}[5,18]=9.126, p<0.01)$. Post hoc comparisons of different time points showed that PER2 expression at ZT10 was significantly higher than that at ZT6 $(p<0.05)$, ZT2, ZT14, ZT18 and ZT22 $(p<0.01)$. PER2 expression at ZT2 was significantly higher than that at ZT18 and ZT22 $(p<0.05)$. The expression at ZT6 was significantly higher than that at ZT18 $(p<0.01)$ and ZT22 $(p<0.05)$. PER2 expression at ZT10 reached a peak, and became the lowest level at ZT18 (Figure 2).

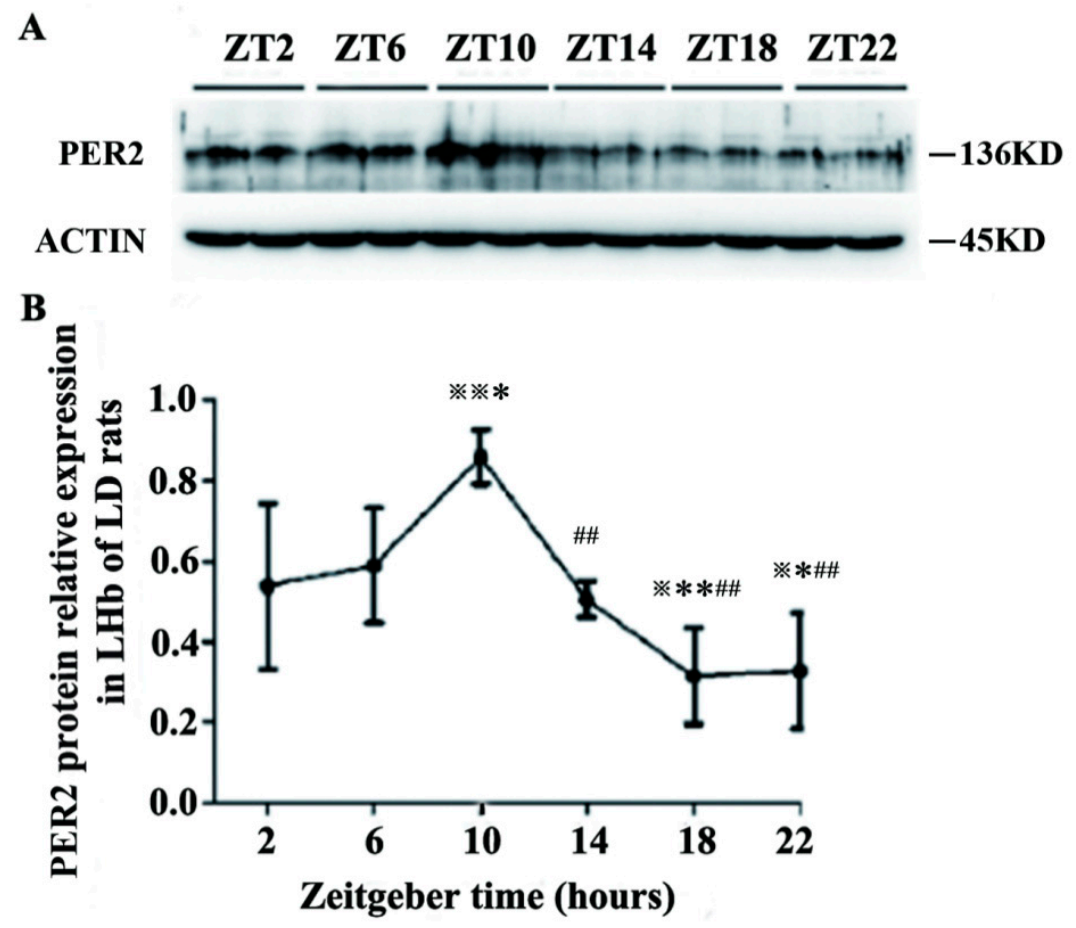

Figure 2. Rhythmic expression of PER2 protein in the LHb of LD rats. (A) Western blotting of PER2 and $\beta$-actin bands in the LHb at six ZT time points; and (B) Relative density of PER2 bands was normalized against $\beta$-actin bands at six ZT time points. Data are presented as mean $\pm \operatorname{SD}(n=6) .{ }^{*} p<0.05$ and ${ }^{* *} p<0.01$ compared with ZT2 time point; $* p<0.05$ and $* * p<0.01$ compared with ZT6 time point; ${ }^{\#} p<0.01$ compared with ZT10 time point.

\subsection{Discussion}

The molecular mechanism of circadian rhythm formation depends on the feedback loop of auto-excitation oscillation from biological clock genes and their protein production [26,27]. Among them, Per2 plays an important role in the circadian rhythmic system [28-30]. In the current study, Per2 expression at the mRNA and protein levels exhibited rhythmic oscillations, with high expression in the light phase and low expression in the dark phase in the LHb under LD conditions. Per2 expression in the LHb reached a peak at ZT10 and a trough at ZT22 (Per2 mRNA) and ZT18 (PER2 protein). Per2 expression at ZT6 was also similar to that at ZT10. These results are consistent 
with the rhythmicity of $\mathrm{LHb}$ neuronal activity that was observed in our previous electrophysiological recordings, which showed that the firing rates of LHb neurons were higher during the day than during the night [22]. Sakhi's research also showed that the firing rates in LHb brain slices was lower in the early day and higher later in the day [31].These results suggest that there might be functional relevance between circadian oscillations of Per2 gene/protein expression at the molecular level and oscillations in the firing rate at the cellular level in the $\mathrm{LHb}$.

The SCN of the hypothalamus contains a circadian pacemaker that regulates many circadian rhythms in mammals. Electrical activity of SCN neurons and Per2 mRNA/protein expression in the SCN have a circadian rhythm, which is high during the day and low at night [32-34]. Rhythmic activity in $\mathrm{LHb}$ neurons at cellular and molecular levels coincide with that in the SCN, suggesting that $\mathrm{LHb}$ activity might be controlled by the SCN. Similarly, Guilding showed that the peak phase of Per2 expression was not correlated with zeitgeber time in the LHb mouse brain in vitro when they used video microscopy imaging and photon-counting of a Per2: luciferase fusion protein [24]. This result indicated that the $\mathrm{LHb}$ cannot maintain stabilization of the oscillation phase and synchronization of neuronal activity in vitro, when they are devoid of any input from the SCN or other known circadian oscillators. The major output from the SCN includes projections of vasoactive intestinal peptide and arginine vasopressin neurons [35]. Interestingly, the LHb receives a vasopressin-containing projection directly from the SCN, and this plays an important role in conveying circadian information $[25,36]$. Therefore, rhythmic expression of Per2 gene/protein in the LHb in vivo might be regulated by the vasopressin pathway from $\mathrm{SCN}$ to $\mathrm{LHb}$ to maintain synchronization with SCN rhythms. Further evidence has shown that the SCN has remarkable asymmetry of c-Fos expression in "splitting" hamsters that are maintained in constant illumination. An obvious left-right asymmetry of c-Fos expression was also found in the medial division of the LHb during the active phase [23]. In addition, the $\mathrm{SCN}$ and $\mathrm{LHb}$ receive a direct retinal projection that conveys light information and responds to light illumination input to induce rhythmic gene expression [22,37-39]. This would explain why rhythmic expression of Per2 gene/protein in the LHb is synchronized with that of the SCN in vivo.

Many physiological functions that are controlled by the LHb show circadian rhythmicity and the $\mathrm{LHb}$ has been shown to generate firing rate rhythms in vivo and in vitro. These findings at the molecular level demonstrate that the $\mathrm{LHb}$ in vivo has diurnal expression of Per2 gene/protein in the LD condition. However, how the LHb plays a role in the circadian rhythm system needs to be further investigated.

\section{Experimental Section}

\subsection{Animals}

Male Wistar rats (100-120 g) were provided by the Center for Experimental Animals, Jilin University, Changchun, China (Certification No. SCXK(Ji)2007-0003). Rats were housed in a standard cage with food and water available ad libitum, and kept under a standard condition (temperature, $23 \pm 2{ }^{\circ} \mathrm{C}, 12$-h light/12-h dark cycles, lights on at 7:00 a.m.) for 1 week. Rats were then transferred into individual cages equipped with a $30-\mathrm{cm}$ diameter running wheel for 3 weeks. The light 
phase intensity was 400-500 lux and was $35 \mathrm{~cm}$ above the cage-top level provided by 8 Watt white fluorescent tubes. There was no light in the room during the dark phase.

Wheel-running activity was recorded using ClockLab data collection and analysis software (Coulbourn Instruments, Actimetrics, Allentown, PA, USA). According to the running-wheel activity only rats that had a good ability of entrainment were chosen for sample collection. All experiments were conducted in accordance with international standards of animal welfare, and were approved by the Institutional Committee for Animal Care Research of Jilin University, Changchun, China.

In LD conditions, the time point of ZT12 was when the lights were turned off and the time point of ZT0 was when the lights were turned on. Rats were anesthetized at six different time points in LD conditions (ZT2, ZT6, ZT10, ZT14, ZT18, and ZT22). The brain was immediately removed and then transferred to ice-cold artificial cerebrospinal fluid at $\mathrm{pH}$ 7.4. Two $500 \mu \mathrm{m}$ thick coronal slices of the lateral habenula were collected with vibration cutting machine (ZQP-86, Shanghai five phase instrument Co., Ltd., Shanghai, China) from Bregma $-3.12-4.20 \mathrm{~mm}$. The boundary between medial and lateral habenula is visualized and its distance to midline changes slightly about $10-80 \mu \mathrm{m}$ along habenular rostrocaudal axis. Because the area from the boundary to midline (medial habenula area) is larger in rostral and caudal side of habenular rostrocaudal axis than middle part of that, we dissected lateral habenula with a thin needle from the rostral and caudal side respevtively to avoid the pollution of the medial habenular tissue. The samples were immediately stored at $-80{ }^{\circ} \mathrm{C}$ until total RNA and protein could be extracted for real-time PCR and western blot experiments. All operations were performed under red light with 8-10 lux during the dark phase to avoid the effect of light.

\subsection{Detection of Real-Time PCR}

Total RNA from LHb tissue was extracted using TRIZOL reagent according to the manufacturer's protocol. A total of $1 \mu \mathrm{g}$ RNA was then reverse transcripted to cDNA for quantification of real-time PCR. Real-time-PCR was performed using an SDS 7300 system real-time PCR instrument using Transtart ${ }^{\mathrm{TM}}$ Green $\mathrm{qPCR}$ Super Mix (Beijing Transgen Biotech Limited Corporation, Beijing, China) with a total reaction volume of $20 \mu \mathrm{L}$ in accordance with the manufacturer's instructions. Thermocycling parameters were $95{ }^{\circ} \mathrm{C}$ for $30 \mathrm{~s}$, followed by 40 cycles of $95{ }^{\circ} \mathrm{C}$ for $5 \mathrm{~s}, 55^{\circ} \mathrm{C}$ for $15 \mathrm{~s}$, and $72{ }^{\circ} \mathrm{C}$ for $30 \mathrm{~s}$. The primers were as follows: Per 2 gene sense primer, AGC AAC ACC ACC TTT CAC AA and antisense primer, CGT AGG CTT AGA CCA CCA TC; and $\beta$-actin gene sense primer, CGT AAA GAC CTC TAT GCC AAC A and antisense primer, TAG GAG CCA GGG CAG TAA TC. SDS 7300 system software was used for recording and analysis of real-time PCR data. According to the $C_{\mathrm{t}}$ values of Per 2 and $\beta$-actin of each sample, we analyzed the Per 2 relative content of each sample with the $2^{-\Delta \Delta C t}$ method.

\subsection{Western Blotting Experiments}

$\mathrm{LHb}$ tissues were dissected and total protein of LHb tissues was extracted with radio immunoprecipitation assay (RIPA) containing Phenylmethanesulfonyl fluoride (PMSF). Protein concentrations were determined with BCA protein assay kits. Equal amounts of protein (10 $\mu \mathrm{g}$ for each sample) were electrophoresed on $8 \%$ sodium dodecyl sulfate polyacrylamide gel electrophoresis and transferred to a polyvinylidene difluoride membrane with constant voltage of $15 \mathrm{~V}$ for 25 min. 
Nonspecific reactivity was blocked in 5\% nonfat dry milk in TBST for $2 \mathrm{~h}$ at room temperature. The membrane was then incubated with primary antibodies of Per2 rabbit anti-rat polyclonal antibody (1:1000, Millipore Biological Corporation, Billerica, MA, USA) and $\beta$-actin rabbit anti-rat antibody (1:1000, Cell Signaling Technology, Danvers, MA, USA) overnight at $4{ }^{\circ} \mathrm{C}$. After washing with $1 \times$ TBST for $5 \mathrm{~min}$ three times, the membrane was incubated with HRP-linked goat anti-rabbit IgG antibody (1:1000, Cell Signaling Technology) for $2 \mathrm{~h}$ at room temperature. After 5 min of washing the membrane with $1 \times$ TBST three times, ECL luminescence liquid (Beijing Apply Gene Technology Company, Beijing, China) was added to the membrane's surface to detect the target bands. Finally, the membrane was placed in a luminescent imaging machine for photographs (Gene Gnome; Sygene Bio Imaging, Cambridge, UK) and the target bands were analyzed with a gel imaging system (Tanon Gis-2008, Shanghai, China).

\subsection{Statistical Analysis}

Statistical analyses were conducted with SPSS 17.0 software (SPSS Inc., Chicago, IL, USA). Results are expressed as mean \pm SD. Multigroup data were analyzed by using one-way ANOVA. The post hoc analysis was then conducted to compare the differences of every two time points using Fisher's least significant differences (LSD). Probability values $<0.05$ were considered statistically significant.

\section{Conclusions}

Our study shows that Per2 expression at the mRNA and protein levels in the LHb under LD conditions has diurnal rhythmic oscillations, with high expression during the day and low expression during the night. Our findings will provide a basis for further revealing the role of the LHb in the circadian rhythm system.

\section{Acknowledgments}

This research was supported by the National Natural Science Foundation of China (Nos. 30570579, 81271465 and 81000570$)$.

\section{Author Contributions}

Hua Zhao, Zhigong Zhao, and Haiyan Xu designed the project and wrote the paper; Zhigong Zhao and Haiyan Xu conducted the experiments; and Hua Zhao, Yongmao Liu, Li Mu, and Jinyu Xiao provided technical assistance and materials. All authors read and approved the final manuscript.

\section{Conflicts of Interest}

The authors declare no conflict of interest. 


\section{References}

1. Moore, R.Y.; Eichler, V.B. Loss of a circadian adrenal corticosterone rhythm following suprachiasmatic lesions in the rat. Brain Res. 1972, 42, 201-206.

2. Moore, R.Y. Organization and function of a central nervous system circadian oscillator: The suprachismatic hypothalamic nucleus. Fed. Proc. 1983, 42, 2783-2789.

3. Hastings, M.H.; Brancaccio, M.; Maywood, E.S. Circadian pacemaking in cells and circuits of the suprachiasmatic nucleus. J. Neuroendocrinol. 2014, 26, 2-10.

4. Stephan, F.K.; Zucker, I. Circadian rhythms in drinking behavior and locomotor activity of rats are eliminated by hypothalamic lesions. Proc. Natl. Acad. Sci. USA 1972, 69, 1583-1586.

5. Johnson, R.F.; Moore, R.Y.; Morin, L.P. Loss of entrainment and anatomical plasticity after lesions of the hamster retinohypothalamic tract. Brain Res. 1988, 460, 297-313

6. Golombek, D.A.; Rosenstein, R.E. Physiology of circadian entrainment. Physiol. Rev. 2010, 90, 1063-1102.

7. Moldavan, M.G.; Allen, C.N. Retinohypothalamic tract synapses in the rat suprachiasmatic nucleus demonstrate short-term synaptic plasticity. J. Neurophysiol. 2010, 103, 2390-2399.

8. Abe, M.; Herzog, E.D.; Yamazaki, S.; Straume, M.; Tei, H.; Sakaki, Y.; Menaker, M.; Block, G.D. Circadian rhythms in isolated brain regions. J. Neurosci. 2002, 22, 350-356.

9. Guilding, C.; Piggins, H.D. Challenging the omnipotence of the suprachiasmatic timekeeper: Are circadian oscillators present throughout the mammalian brain? Eur. J. Neurosci. 2007, 25, 3195-3216.

10. Guilding, C.; Hughes, A.T.; Brown, T.M.; Namvar, S.; Piggins, H.D. A riot of rhythms: Neuronal and glial circadian oscillators in the mediobasal hypothalamus. Mol. Brain 2009, 2, doi:10.1186/ 1756-6606-2-28.

11. Lecourtier, L.; Kelly, P.H. A conductor hidden in the orchestra? Role of the habenular complex in monoamine transmission and cognition. Neurosci. Biobehav. Rev. 2007, 31, 658-672.

12. Geisler, S.; Trimble, M. The lateral habenula: No longer neglected. CNS Spectr. 2008, 13, 484-489.

13. Hikosaka, O. The habenula: From stress evasion to value-based decision-making. Nat. Rev. Neurosci. 2010, 11, 503-513.

14. Wagner, C.K.; Silverman, A.J.; Morrell, J.I. Evidence for estrogen receptor in cell nuclei and axon terminals within the lateral habenula of the rat: Regulation during pregnancy. J. Comp. Neurol. 1998, 392, 330-342.

15. Heldt, S.A.; Ressler, K.J. Lesions of the habenula produce stress-and dopamine-dependent alterations in prepulse inhibition and locomotion. Brain Res. 2006, 1073-1074, 229-239.

16. Aizawa, H.; Yanagihara, S.; Kobayashi, M.; Niisato, K.; Takekawa, T.; Harukuni, R. The synchronous activity of lateral habenular neurons is essential for regulating hippocampal theta oscillation. J. Neurosci. 2013, 33, 8909-8921.

17. Yang, L.M.; Hu, B.; Xia, Y.H.; Zhang, B.L.; Zhao, H. Lateral habenula lesions improve the behavioral response in depressed rats via increasing the serotonin level in dorsal raphe nucleus. Behav. Brain Res. 2008, 188, 84-90.

18. McCathy, M.J.; Welsh, D.K. Cellular circadian clocks in mood disorders. J. Biol. Rhythm. 2012, 27, 339-352. 
19. Tavakoli.-Nezhad, M.; Schwartz, W.J. Hamsters running on time: Is the lateral habenula a part of the clock? Chronobiol. Int. 2006, 23, 217-224.

20. Paul, M.J.; Indic, P.; Schwartz, W.J. A role for the habenula in the regulation of locomotor activity cycles. Eur. J. Neurosci. 2011, 34, 478-488.

21. Zhao, H.; Zhang, B.L.; Yang, S.J.; Rusak, B. The role of lateral habenula-dorsal raphe nucleus circuits in higher brain functions and psychiatric illness. Behav. Brain Res. 2015, 277, 89-98.

22. Zhao, H.; Rusak, B. Circadian firing-rate rhythms and light responses of rat habenula nucleus neurons in vivo and in vitro. Neuroscience 2005, 132, 519-528.

23. Tavakoli-Nezhad, M.; Schwartz, W.J. c-fos expression in the brains of behaviorally "split" hamsters in constant light: Calling attention to a dorsolateral region of the suprachiasmatic nucleus and the medial division of the lateral habenula. J. Biol. Rhythm. 2005, 20, 419-429.

24. Guilding, C.; Hughes, A.T.; Piggins, H.D. Circadian oscillators in the epithalamus. Neuroscience 2010, 169, 1630-1639.

25. Buijs, R.M. Intraand extrahypothalamic vasopressin and oxytocin pathways in the rat. Pathways to the limbic system, medulla oblongata and spinal cord. Cell Tissue Res. 1978, 192, 423-435.

26. Takahashi, J.S.; Hong, H.K.; Ko, C.H.; McDearmon, E.L. The genetics of mammalian circadian order and disorder: Implications for physiology and disease. Nat. Rev. Genet. 2008, 9, 764-775.

27. Lowrey, P.L.; Takahashi, J.S. Genetics of circadian rhythms in Mammalian model organisms. Adv. Genet. 2011, 74, 175-230.

28. Zheng, B.; Larkin, D.W.; Albrecht, U.; Sun, Z.S.; Sage, M.; Eichele, G.; Lee, C.C.; Bradley, A. The mPer2 gene encodes a functional component of the mammalian circadian clock. Nature 1999, 400, 169-173.

29. Bae, K.; Jin, X.; Maywood, E.S.; Hastings, M.H.; Reppert, S.M.; Weaver, D.R. Differential functions of mPer1, mPer2, and mPer3 in the SCN circadian clock. Neuron 2001, 30, 525-536.

30. Pendergast, J.S.; Friday, R.C.; Yamazaki, S. Distinct functions of Period2 and Period3 in the mouse circadian system revealed by in vitro analysis. PLOS ONE 2010, 5, e8552.

31. Sakhi, K.; Wegner, S.; Belle, M.D.C.; Howarth, M.; Delagrange, P.; Brown, T.M.; Piggins, H.D. Intrinsic and extrinsic cues regulate the daily profile of mouse lateral habenula neuronal activity. J. Physiol. 2014, 592, 5025-5045.

32. Vasalou, C.; Henson, M.A. A Multiscale Model to Investigate Circadian Rhythmicity of Pacemaker Neurons in the Suprachiasmatic Nucleus. PLoS Comput. Biol. 2010, 6, e1000706.

33. Carr, A.J.; Johnston, J.D.; Semikhodskii, A.G.; Nolan, T.; Cagampang, F.R.; Stirland, J.A.; Loudon, A.S. Photoperiod differentially regulates circadian oscillators in central and peripheral tissues of the syrian hamster. Curr. Biol. 2003, 13, 1543-1548.

34. Rocha, V.A.; Frazão, R.; Campos, L.M.; Mello, P.; Donato, J.J.; Cruz-Rizzolo, R.J.; Nogueira, M.I.; Pinato, L. Intrinsic organization of the suprachiasmatic nucleus in the capuchin monkey. Brain Res. 2014, 1543, 65-72.

35. Watts, A.G.; Swanson, L.W.; Sanchez-Watts, G. Efferent projections of the suprachiasmatic nucleus: I. Studies using anterograde transport of Phaseolus vulgaris leucoagglutinin in the rat. J. Comp. Neurol. 1987, 258, 204-229.

36. Sofroniew, M.V.; Weindl, A. Projections from the parvocellular vasopressin and neurophysin-containing neurons of the suprachiasmatic nucleus. Am. J. Anat. 1978, 153, 391-429. 
37. Qu, T.; Dong, K.; Sugioka, K.; Yamadori, T. Demonstration of direct input from the retina to the lateral habenular nucleus in the albino rat. Brain Res. 1996, 709, 251-258.

38. Reuss, S.; Decker, K. Anterograde tracing of retinohypothalamic afferents with Fluoro-Gold. Brain Res. 1997, 745, 197-204.

39. Hannibal, J.; Kankipati, L.; Strang, C.E.; Peterson, B.B.; Dacey, D.; Gamlin, P.D. Central projections of intrinsically photosensitive retinal ganglion cells in the macaque monkey. J. Comp. Neurol. 2014, 522, 2231-2248.

(C) 2015 by the authors; licensee MDPI, Basel, Switzerland. This article is an open access article distributed under the terms and conditions of the Creative Commons Attribution license (http://creativecommons.org/licenses/by/4.0/). 